\title{
Dynamic Tracking Human Mesenchymal Stem Cells Tropism following Smoke Inhalation Injury in NOD/SCID Mice
}

\author{
MeiJuan Song, ${ }^{1}$ Qi Lv, ${ }^{2}$ XiuWei Zhang, ${ }^{1}$ Juan Cao, ${ }^{2}$ ShuLi Sun, ${ }^{2}$ PeiXin Xiao, \\ ShiKe Hou, ${ }^{2}$ Hui Ding, ${ }^{2}$ ZiQuan Liu, ${ }^{2}$ WenLong Dong, ${ }^{2}$ JinQiang Wang, ${ }^{2}$ Xue Wang, \\ ZhiGuang Sun, ${ }^{2}$ Man Tian, ${ }^{3}$ and HaoJun Fan ${ }^{2}$ \\ ${ }^{1}$ Respiratory Department, Affiliated Jiangning Hospital, Nanjing Medical University, Jiangsu, China \\ ${ }^{2}$ Institute of Disaster Medicine and Public Health, Affiliated Hospital of Logistic University of Chinese People's Armed Police Force, \\ Tianjin 300162, China \\ ${ }^{3}$ Respiratory Department, Affiliated Nanjing Children's Hospital, Nanjing Medical University, Jiangsu, China
}

Correspondence should be addressed to Man Tian; tmsweet@163.com and HaoJun Fan; haojunfan86@163.com

Received 21 March 2016; Revised 22 June 2016; Accepted 28 June 2016

Academic Editor: Francesco Petrella

Copyright (c) 2016 MeiJuan Song et al. This is an open access article distributed under the Creative Commons Attribution License, which permits unrestricted use, distribution, and reproduction in any medium, provided the original work is properly cited.

\begin{abstract}
Multiple preclinical evidences have supported the potential value of mesenchymal stem cells (MSCs) for treatment of acute lung injury (ALI). However, few studies focus on the dynamic tropism of MSCs in animals with acute lung injury. In this study, we track systemically transplanted human bone marrow-derived mesenchymal stem cells (hBMSCs) in NOD/SCID mice with smoke inhalation injury (SII) through bioluminescence imaging (BLI). The results showed that hBMSCs systemically delivered into healthy NOD/SCID mouse initially reside in the lungs and then partially translocate to the abdomen after $24 \mathrm{~h}$. Compared with the uninjured control group treated with hBMSCs, higher numbers of hBMSCs were found in the lungs of the SII NOD/SCID mice. In both the uninjured and SII mice, the BLI signals in the lungs steadily decreased over time and disappeared by 5 days after treatment. hBMSCs significantly attenuated lung injury, elevated the levels of KGF, decreased the levels of TNF- $\alpha$ in BALF, and inhibited inflammatory cell infiltration in the mice with SII. In conclusion, our findings demonstrated that more systemically infused hBMSCs localized to the lungs in mice with SII. hBMSC xenografts repaired smoke inhalation-induced lung injury in mice. This repair was maybe due to the effect of anti-inflammatory and secreting KGF of hMSCs but not associated with the differentiation of the hBMSCs into alveolar epithelial cells.
\end{abstract}

\section{Introduction}

Smoke inhalation injury (SII) is caused by smoke-induced damage of the respiratory tract and lung parenchyma, with or without additional heat-induced damage. SII is a major cause of morbidity and mortality in victims of fire tragedies [1], affecting approximately $22 \%$ of all burn patients and resulting in at least $30 \%$ of all fire-related mortality [2]. Moreover, $80 \%$ to $90 \%$ of fire-related fatalities have been attributed to smoke inhalation [3]. The major harmful components of smoke include heat, systemic toxins (e.g., $\mathrm{CO}$ and cyanide), and respiratory irritants [4], which damage the respiratory tract and lung tissue. This results in laryngeal/pulmonary edema, airway obstruction, and ventilation/perfusion mismatch [5].
Severe cases may develop acute respiratory distress syndrome (ARDS) [3]. Determining the best method for treating burn victims, especially during the early stages of smoke inhalation-induced acute lung injury (ALI), remains to be a difficult problem in the field of first aid medicine. Current treatments for SII mainly focus on oxygen administration, airway management, fluid resuscitation, mechanical ventilation, and the use of specific medications $[1,4,6]$. Although many drugs are effective in reducing lung injury in animal models, only a few drugs, including anticoagulants, $\beta 2$ agonists, antioxidants, and inflammatory mediator agonists, are currently applied in the clinical setting $[6,7]$.

Mesenchymal stem cells (MSCs) are self-renewing, multipotent progenitor cells that have the potential to differentiate 
into multiple different mesodermal lineages. It has been shown in many different animal models that MSCs have a remarkable ability to localize to sites of injury and exert nonimmunogenic and immunosuppressive characteristics $[8,9]$. Based on these properties, MSCs offer a promising source for cell-based treatment of various complicated disorders, such as graft-versus-host disease [10,11], cardio/cerebrovascular disease [12], spinal cord injury [13], hepatic disease [14], and respiratory disease $[15,16]$. Furthermore, many studies have indicated that MSCs exert protective effects against ALI via their secretion of multiple paracrine factors, including endothelial and epithelial growth factors, anti-inflammatory cytokines, and antimicrobial peptides [17-20]. However, these studies have mainly focused on endotoxin-induced ALI, and researches focusing on the effects of MSCs on smoke inhalation-induced ALI still lack. Furthermore, uncertainties remain regarding the localization and persistence of MSCs in vivo following their administration into subjects with ALI.

Bioluminescent imaging (BLI), a recently developed technique that enables the noninvasive study of ongoing biological processes in small laboratory animals, can be used to track luciferase- (Luc-) expressing cells implanted into living animals in real time. In a previous study, Kidd et al. used BLI to track the dynamic distribution of firefly Lucexpressing human MSCs (hMSCs) following their systemic injection into healthy mice, mice subjected to inflammatory insults, and mice bearing tumors. The hMSCs were found to initially localize to the lungs and later moved into the liver and spleen. Additionally, the Luc signal produced by the hMSCs decreased over time. In wounded mice and tumor-bearing mice, the hMSCs were found to localize to injured tissue or tumors after systemic administration [21]. Although it has been shown that MSCs initially localize to the lungs following systemic delivery, studies examining the dynamic distribution of MSCs after their intravenous injection into mice with SII are lacking. In the current study, we modified human bone marrow-derived MSCs (hBMSCs) to stably coexpress Luc and green fluorescent protein (GFP) reporter genes (Luc-GFP-hBMSCs). We then used BLI to track the dynamics of the cells' localization patterns for 14 days following their systemic administration into normal mice and mice with SII. Our results provide experimental support for the use MSCs to treat SII.

\section{Materials and Methods}

2.1. Cells and Animals. hMSCs were purchased from Cyagen Biosciences (Guangzhou, China) and grown in Dulbecco's modified Eagle's medium (DMEM) (Cyagen Biosciences) supplemented with $10 \%$ heat-inactivated fetal bovine serum (FBS) (Cyagen) at $37^{\circ} \mathrm{C}$ under $5 \% \mathrm{CO}_{2}$.

Male NOD/SCID mice, aged between 6 and 8 weeks and ranging in weight from 25 to $30 \mathrm{~g}$, were purchased from Wei Tong Li Hua Experimental Animal Technology Co., Ltd (Beijing, China). The mice were used in accordance with institutional guidelines and following approved protocols.

2.2. Lentiviral Vector Construction and MSC Transduction. Lentiviral vectors carrying a Luc and GFP dual-fusion reporter gene were constructed and purified by Shanghai GeneChem Co., Ltd. For transduction, hMSCs were seeded into $25 \mathrm{~cm}^{2}$ flasks containing appropriate growth medium and grown to $20 \%-30 \%$ confluence. Then, the GFP-Luc lentivirus vectors were added at a multiplicity of infection (MOI) of 10 to a $2.5 \mathrm{~mL}$ aliquot of hMSCs in growth medium containing $5 \mu \mathrm{g} / \mathrm{mL}$ polybrene. The cells were incubated with the viruses for $8-12 \mathrm{~h}$, after which fresh medium was added to each flask, and the cells were incubated for an additional 48-72 h. The cells were passaged 1:2 and grown to 80$100 \%$ confluence. Three days after transduction, the cells were viewed on a Leica DMI4000 inverted microscope equipped with a fluorescence source and a charge-coupled device (CCD) camera. Transduction efficiency was determined by fluorescence-activated cell sorting (FACS) analysis of GFP expression using previously described settings [22].

2.3. Flow Cytometry Analysis. Luc-GFP-hBMSCs were harvested with $0.25 \%$ trypsin-EDTA and resuspended in phosphate-buffered saline (PBS) supplemented with 2\% FBS. Approximately $1 \times 10^{6}$ cells were stained with $1 \mu \mathrm{g}$ of antibody for 30 minutes at $4^{\circ} \mathrm{C}$ and then analyzed on a FACS Caliber flow cytometer (Becton Dickinson, Franklin Lakes, NJ). Human antibodies against the following proteins were used for this analysis: CD105, CD29, CD73, CD44, CD90, CD34, CD45, and CD11c (BD Biosciences).

2.4. Multilineage Differentiation of Transduced hMSCs. To determine the multilineage differentiation potential of the transduced hMSCs, we cultured the cells in various types of differentiation media according to manufacturer recommendations (Cyagen Biosciences, Guangzhou, China, http:// www.cyagen.com/). To induce adipogenic differentiation, Luc-GFP-hBMSCs were subcultured in six-well plates at 2 $\times 10^{4}$ cells $/ \mathrm{cm}^{2}$ in growth medium containing $10 \%$ FBS, $5 \%$ penicillin-streptomycin, and $2 \mathrm{mM} \mathrm{L}$-glutamine. The culture medium was replaced every 3 days until the cells reached $100 \%$ confluence, after which the growth medium was replaced with induction medium ( $2 \mathrm{~mL}$ per well) containing FBS, penicillin-streptomycin, glutamine, insulin, rosiglitazone, and dexamethasone. Three days later, the medium was replaced with maintenance medium consisting of FBS, penicillin-streptomycin, and insulin. After $24 \mathrm{~h}$, the maintenance medium was changed back to induction medium, and this cycle was repeated three times. After five cycles of induction/maintenance, the cells were cultured in maintenance medium for 3 days. Three weeks later, adipose cells were stained for visualization with Oil Red O.

To induce osteogenic differentiation, Luc-GFP-hBMSCs were cultured in growth medium at a density of $3 \times$ $10^{4}$ cells $/ \mathrm{cm}^{2}$ for 1 day at $37^{\circ} \mathrm{C}$ in a $5 \% \mathrm{CO}_{2}$ humidified incubator. Following this, the growth medium was aspirated and replaced with osteogenic differentiation medium ( $2 \mathrm{~mL}$ per well) containing FBS, penicillin-streptomycin, glutamine, ascorbate, $\beta$-glycerophosphate, and dexamethasone. The medium was replaced every three days. Three weeks later, the cells were fixed with $2 \mathrm{~mL}$ of $4 \%$ formaldehyde solution and stained with Alizarin red. A light microscope was used to visualize and capture images of the stained cells. 
To induce chondrogenic differentiation, human MSCs at subconfluent conditions were trypsinized and aliquots of 2 $\times 10^{5}$ cells per well were added to a $15 \mathrm{~mL}$ centrifuge tube, and the plate was spun at $400 \times \mathrm{g}$ for $5 \mathrm{~min}$. For differentiation into chondrocytes, cells were cultured in a commercialized chondrogenic induction medium in the presence of $10 \mathrm{ng} / \mathrm{mL}$ recombinant human TGF- $\beta 3$. The cell pellets formed freefloating aggregates within the first $24 \mathrm{~h}$. The medium was replaced every 2-3 days, and aggregates were cultured for 28 days and collected for paraffin section following Alcian Blue staining.

2.5. Establishment of Smoke Inhalation Mouse Model. All animals used in this study received humane care in compliance with the Guide for the Care and Use of Laboratory Animals published by the National Institutes of Health. The study protocol was approved by the Laboratory Animal Ethics Committee of the Affiliated Hospital of Logistical College of Chinese People's Armed Police Forces. All surgeries were performed under sodium pentobarbital anesthesia, and all efforts were made to minimize suffering.

Models of SII were established using a previously described device that was constructed in-house [23]. To accomplish this, awake male NOD/SCID mice were exposed to combustion smoke generated by smoldering wood shavings in a smoke-generating container connected to a $20 \mathrm{~L}$ transparent exposure chamber. The mice were subjected to the smoke for $0,3,5,7$, and $9 \mathrm{~min}$. The establishment of severe SII was assessed by blood carboxyhemoglobin $(\mathrm{COHb})$ concentration, blood gas analysis, measurement of the wet/dry (W/D) weight ratio of lung tissue, and lung histopathology. Blood was collected from a subset of mice that were killed $1 \mathrm{~h}$ after smoke exposure to measure $\mathrm{COHb}$ concentration with an oximeter (482 CO-Oximeter) [24] and analyze blood gas content using a Radiometer ABL 625 Blood Gas Analyzer (Copenhagen, Denmark) [25]. Another subset of mice were killed $3 \mathrm{~d}$ after smoke exposure, and their lungs were isolated to measure $\mathrm{W} / \mathrm{D}$ ratios and for histological analysis.

2.6. Luc-GFP-hBMSC Administration and Bioluminescent Imaging. At $24 \mathrm{~h}$ after smoke inhalation, $100 \mu \mathrm{L}$ aliquots of Luc-GFP-hBMSCs $\left(3 \times 10^{5}\right.$ cells $)$ were injected into the tail veins of control and SII NOD/SCID mice. The mice were then submitted to BLI to visualize the localization of the Luc-GFPhBMSCs at $1.5,2.5,5,7.5,10$, and $24 \mathrm{~h}$ and 3 and 5 days after injection [21].

To monitor Luc-GFP-hBMSC localization to the lungs, we submitted mice to isoflurane anesthesia and then intraperitoneally injected them with D-luciferin firefly potassium salt substrate (150 mg/kg body weight in $100 \mu \mathrm{L}$ PBS). Then, we placed the animals into an IVIS system in a supine position (Caliper Life Sciences, Hopkinton, MA). The animals were imaged over a $10 \mathrm{~min}$ time period with $1 \mathrm{~min}$ acquisition intervals [26]. To quantify light emission, a region of interest (ROI) was manually selected based on signal intensity. The area of the ROI was kept constant while the signal intensity was recorded as average photons per second per square centimeter per steradian as previously described [21].

2.7. Analysis of Wet/Dry Weight Ratio of Lung Tissue. After treatment as described above, the mice were killed, and their left lungs were isolated. After blotting off blood and other contaminants, the wet weights of the lung tissue samples were measured. Then, the lungs were dried in a $70^{\circ} \mathrm{C}$ oven for $72 \mathrm{~h}$, and their dry weights were measured. The W/D weight ratios of the lungs were then calculated as previously described [25].

2.8. Histology and Immunohistochemistry. The right lungs of the mice treated as described above were isolated, and their upper and middle lobes were fixed in $10 \%$ formalin for $24 \mathrm{~h}$. The tissue samples were then dehydrated, embedded in paraffin, and cut into $5 \mathrm{~mm}$ thick sections. Following this, the samples were stained with hematoxylin and eosin (H\&E) after deparaffinization and evaluated under an optical microscope (Olympus BX51, Japan).

Luc-GFP-hBMSCs in lung tissue were detected by immunostaining for GFP. After deparaffinization and rehydration, paraffin sections were placed into a pressure cooker containing antigen retrieval buffer $(0.01 \mathrm{M}$ citrate buffer, $\mathrm{pH}$ 6.0) under full pressure for 2 minutes to unmask antigens. Immunostaining was performed by incubating the sections with a rabbit anti-GFP monoclonal antibody (1:100, Abcam, MA) overnight at $4^{\circ} \mathrm{C}$, followed by incubation with a biotinconjugated secondary antibody (ZSGB-bio, China) at $37^{\circ} \mathrm{C}$ for $1 \mathrm{~h}$ and horseradish peroxidase-conjugated streptavidin (ZSGB-bio, China) at $37^{\circ} \mathrm{C}$ for $30 \mathrm{~min}$. The sections were stained with a DAB kit, which were counterstained with hematoxylin to visualize cell nuclei. Images were obtained with an Olympus BX51 microscope, and the proportion of positively stained cells was determined using Image-Pro Plus version 5.1. For histological and immunohistochemical analysis, the slides were labeled with numbers, and doubleblinded examinations were performed by two independent pathologists.

2.9. Semiquantitative PCR. Total RNA for PCR was extracted using an RNeasy kit (Solarbio, Beijing, China), which included a DNase digestion step to remove any contaminating DNA. Semiquantitative reverse transcription PCR was performed using a thermal cycler (Thermo), and amplified products were visualized using agarose gels. The following primers were used for PCR:

Luciferase forward: ACTGGGACGAAGACGAACAC.

Luciferase reverse: GGCGACGTAATCCACGATCT.

$\beta$-actin forward: GTGGGGCGCCCCAGGCACCA. $\beta$-actin reverse: CTTCCTTAATGTCACGCACGATTTC.

2.10. Analysis of TNF- $\alpha$ and KGF Levels in Bronchoalveolar Lavage Fluid. Bronchoalveolar lavage (BAL) was performed by instilling and withdrawing sterile physiological saline 
$(1 \mathrm{~mL})$ through a tracheal cannula using a 20-gauge Surflo i.v. catheter. This procedure was repeated three times, and the three BAL fluid (BALF) samples were pooled. The BALF was centrifuged $(300 \times \mathrm{g}, 5 \mathrm{~min})$, and the supernatant portions were stored at $-80^{\circ} \mathrm{C}$ for further examination. For the detections of TNF- $\alpha$ and keratinocyte growth factor (KGF), the supernatant of BALF was analyzed by using mouse TNF- $\alpha$ ELISA kit (eBioscience, San Diego, CA, USA) and KGF ELISA kit (Nanjing Jiancheng Bioengineering Institute, Nanjing, China) following the instructions of manufacturer.

2.11. Statistical Analysis. All data were processed using SPSS version 13.0 statistical software. The data are shown as the mean \pm standard deviation $(\bar{x} \pm s)$. Sample measurement data between groups were compared using independent samples $t$-tests, and group data were compared using paired $t$-tests. $P<0.05$ was considered significant.

\section{Results}

3.1. hMSC Transduction. Lenti-GFP-Luc with a $2.00 \mathrm{E}+$ $8 \mathrm{TU} / \mathrm{mL}$ titer was used at an MOI of 10 to infect hMSCs. Transduction efficiency was approximately $90 \%$ after $48 \mathrm{~h}$ based on fluorescence and phase-contrast microscopy (Figures 1(a) and 1(b)). GFP expression remained stable for at least $30 \mathrm{~d}$ under constant culture conditions (data not shown). In vitro Luc activity was assessed using BLI following the application of D-luciferin. Only the transduced cells showed Luc activity and not the control cells (Figure 1(c)).

3.2. Characterization of Luc-GFP-hBMSCs. After transduction with Lenti-GFP-Luc, FACS analysis demonstrated that Luc-GFP-hBMSCs expressed high levels of CD105, CD29, CD73, CD44, and CD90 and low levels of CD34, CD45, and CD11c. These proteins were chosen for analysis because they represent well-established phenotypic markers for hMSCs (Figure 2(a)) [23]. The expression patterns were consistent across all hMSCs tested, and the tested hMSCs were used in the following experiments. Additionally, we subjected transduced hMSCs to adipogenic and osteoblastic differentiation assays. In all cases, cells positively stained for Oil Red $\mathrm{O}$ and Alizarin red were detected after culture in differentiation medium, suggesting that the cells maintained differentiation potential regardless of lentiviral transduction (Figures 2(b) and 2(c)). Furthermore, chondrogenic differentiation of transduced hMSCs was confirmed by staining the acid mucopolysaccharide of chondrocytes with Alcian Blue (Figure 2(d)).

\subsection{Establishment of Mouse Model of Smoke Inhalation Injury.}

To establish a mouse model of SII, a smoke generator constructed in-house was utilized, as previously described [23]. To optimize the experimental conditions for inducing SII, mice were subjected to smoke for $0,3,5,7$, and 9 min. $85 \%$ of mice exposed to smoke for 9 min died of hypoxia, so this time point was excluded in the following detections. $\mathrm{COHb}$ concentration and blood gas content were measured immediately after smoke exposure and again $1 \mathrm{~h}$ later. As the smoke exposure time increased, the $\mathrm{COHb}$ concentration increased, while the $\mathrm{PaO}_{2}$ and $\mathrm{PaO}_{2} / \mathrm{FiO}_{2}$ content in blood decreased (Figure 1(a)). The $\mathrm{PaO}_{2} / \mathrm{FiO}_{2}$ ratio was below 300 at $7 \mathrm{~min}$ in the SII group, which is within the standard for mild ARDS according to the Berlin Definition [27].

The W/D weight ratio was used as an index of water accumulation in the lung, which is an indicator of lung edema. At $3 \mathrm{~d}$ after smoke exposure, the lung $\mathrm{W} / \mathrm{D}$ ratios in the $7 \mathrm{~min}$ SII group were significantly elevated relative to the control group; however, there were no differences noted in the 3 or 5 min SII groups (Figure 3(a)). Accordingly, histopathology results showed that exposure to smoke for $7 \mathrm{~min}$ led to the most serious pathological changes in the lungs, including narrowed alveolar space, thickened alveolar and bronchiole walls, and inflammatory cell infiltration around the airway (Figure 3(b)). Based on these results, we used a 7 min smoke exposure protocol for the following studies.

3.4. Dynamics of hMSC Distribution after Injection into Mice with SII. Luc-GFP-hBMSCs $\left(3 \times 10^{5}\right.$ cells/animal $)$ were intravenously injected into NOD/SCID mice with or without SII. Live-animal BLI was then used to monitor LucGFP-hBMSC localization patterns over time. As shown in Figure 4, luciferase expression was initially detected in the lungs in both the control and the SII plus Luc-hBMSC groups (Figure 4(a)). Compared with the control group, stronger BLI signals were produced in the SII group. The BLI signals in the lungs peaked at $7.5 \mathrm{~h}$ after infusion of hBMSCs and then gradually diminished with time (Figure 4(b)). At $24 \mathrm{~h}$ after infusion of hBMSCs, the bioluminescent signals began to shift to the abdomen in the control group. However, the signals were still primarily in the lungs in the mice with SII. Five days after injection of hBMSCs, the BLI signals disappeared completely from the lungs, and the abdomens of the mice in the control group showed only weak BLI signals. Fourteen days after injection, no signals were detected in either the control group or the SII group (Figures 4(a) and $4(\mathrm{~b}))$.

3.5. PCR and Immunohistochemistry Detection of $h B M S C$ Engraftment. Fourteen days after the injection of Luc-GFPhBMSCs, the injected mice were sacrificed, and PCR was used to detect Luc expression in liver and lung tissues. Consistent with the BLI results described above, no Luc expression was detected in either the control group or the SII group that underwent hBMSC administration (Figure 5(a)). Accordingly, no GFP-positive cells were found in the lung tissue samples collected from these mice (Figure 5(b)).

3.6. Protective Effects of hBMSC Xenografts in SII Mice. Fourteen days after the injection of hBMSCs into mice with SII, we evaluated the effects produced by the cells by measuring lung W/D weight ratios and evaluating pathological changes in lung tissue samples. There were no significant differences in W/D weight ratio in the control group before and after injection. The lung W/D ratio in the SII + hBMSC group was significantly lower than that in the untreated SII group $(P<0.01)$, indicating that pretreatment with hBMSCs could decrease the degree of lung edema produced by smoke inhalation (Figure 6(a)). Moreover, $\mathrm{PaO}_{2}$ and $\mathrm{PaO}_{2} / \mathrm{FiO}_{2}$ were 

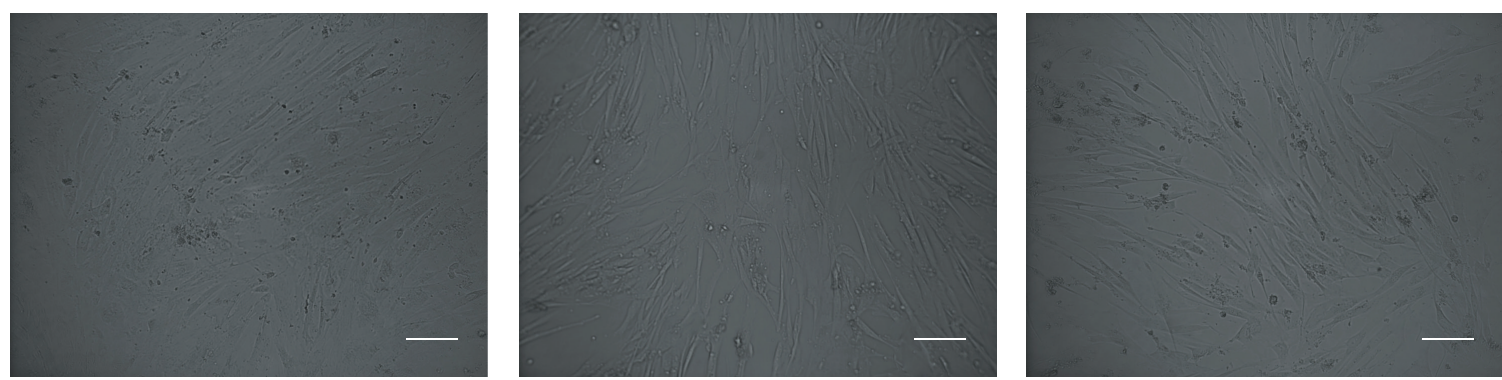

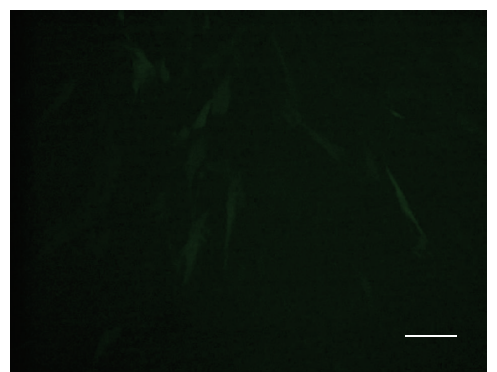

MOI $=10$

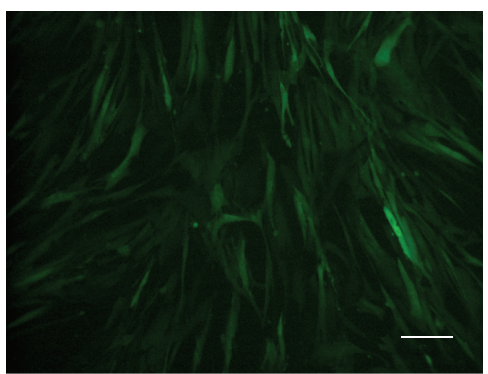

MOI $=10+$ polybrene

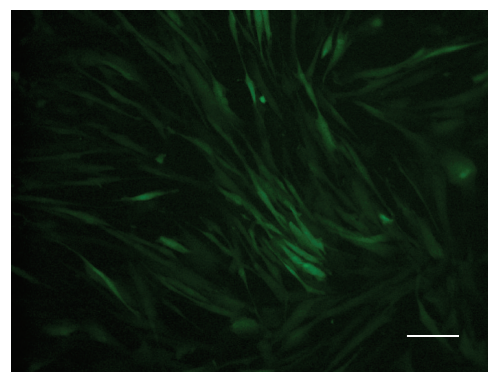

MOI $=50+$ polybrene

(a)

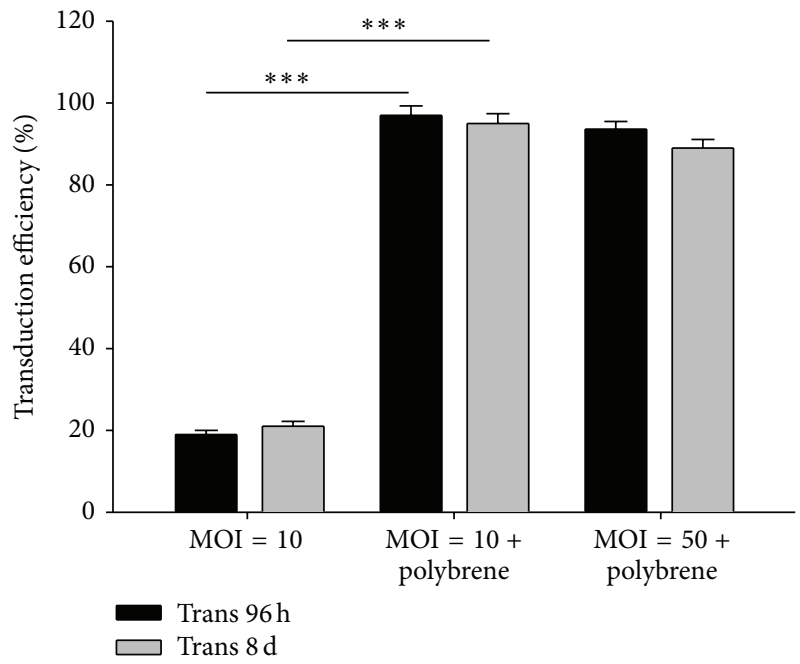

(b)

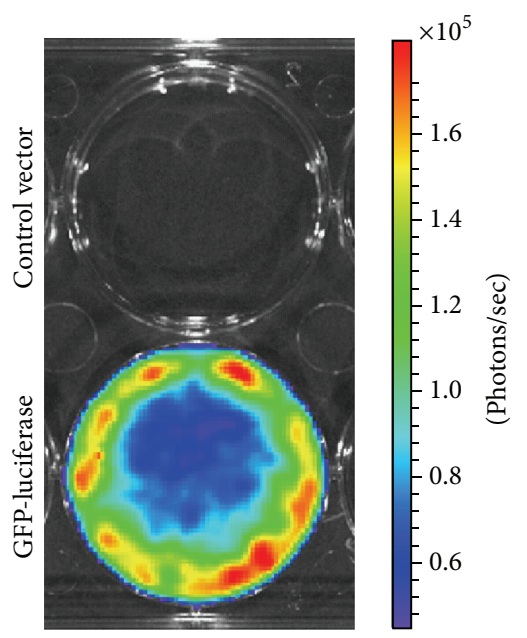

(c)

FIGURE 1: Characterization of human mesenchymal stem cells expressing firefly luciferase and green fluorescent protein reporter genes. (a) Human mesenchymal stem cells (hMSCs) were transduced with a lentiviral vector carrying luciferase (Luc) and green fluorescent protein (GFP) reporter genes, and GFP expression within the cytosol was measured. (b) Transduction efficiencies of Luc-GFP lentiviral vectortransduced hMSCs after $96 \mathrm{~h}$ and $8 \mathrm{~d} . * * *$ indicates $P<0.01$ between the indicated groups. (c) Firefly Luc-expressing cells showed specific activity after treatment with D-luciferin and coelenterazine in vitro.

significantly improved after hBMSCs treatment compared with SII group $(P<0.01)$ (Figures 6(b) and 6(c)).

As shown in Figure 6(d), there were no obvious differences in lung tissue samples collected from mice treated with hBMSCs compared to untreated mice. In the SII group, the alveolar walls burst, and the alveolar space was narrowed. In addition, there was significant infiltration of polymorphonuclear leukocytes (PMNs) around the airway. The administration of hBMSCs markedly reduced the severity of pulmonary injury induced by smoke inhalation. In the lungs of the mice in the SII + hBMSCs group, the alveolar space was widened, there was less PMN infiltration, and there were thinner alveolar septa compared to the lungs of the untreated mice with SII. These results indicated that the hBMSC xenografts protected mice from damage associated with SII.

3.7. Analysis of KGF and TNF- $\alpha$ Levels in BALF. Previous studies have reported that MSCs could repair ALI-induced impaired alveolar fluid clearance (AFC) by secreting KGF [20]. We next measured the concentration of KGF in BALF and culture supernatant of hBMSCs. Consistent with several studies, the secret of KGF by hBMSCs was detected in 

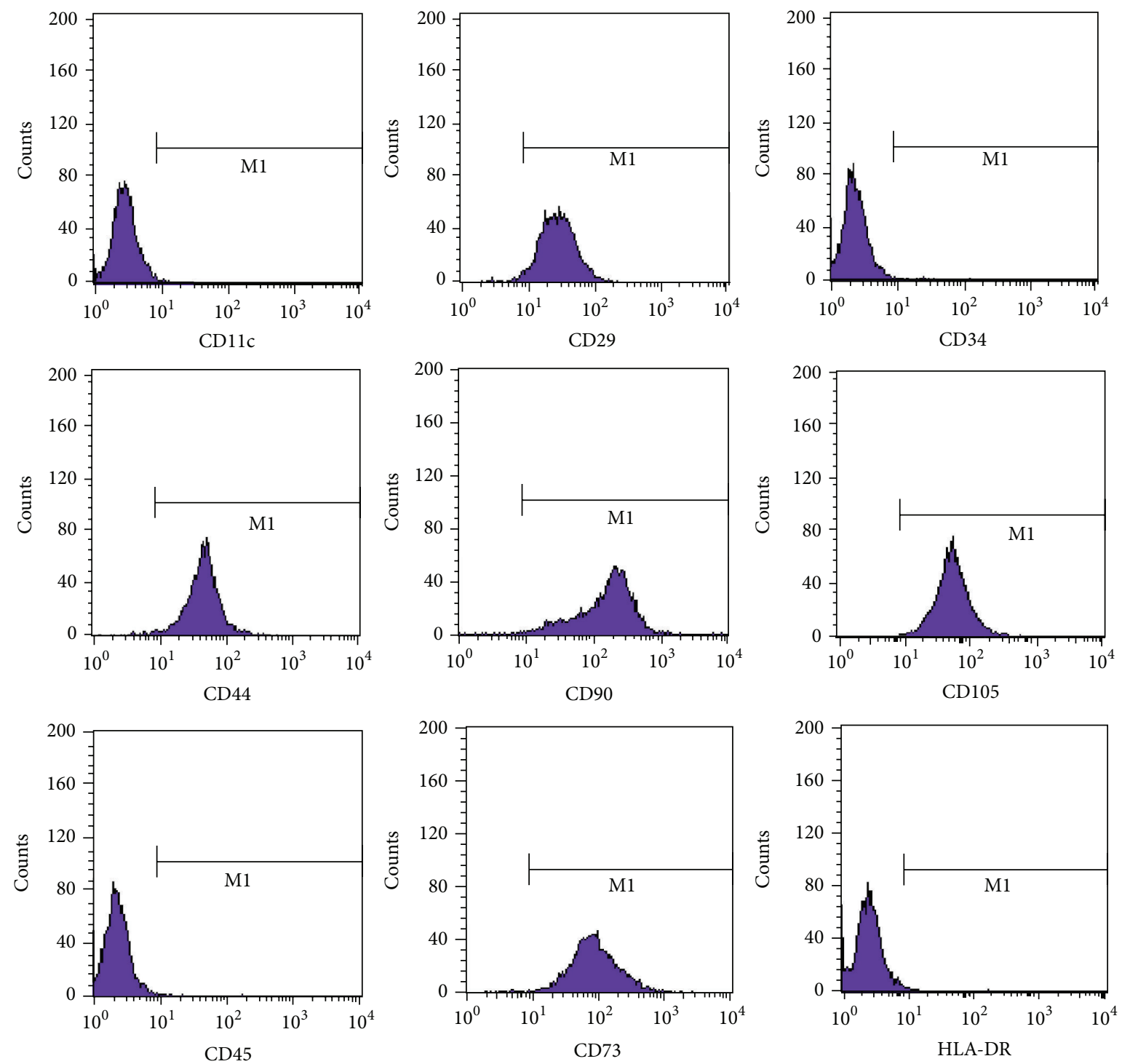

(a)

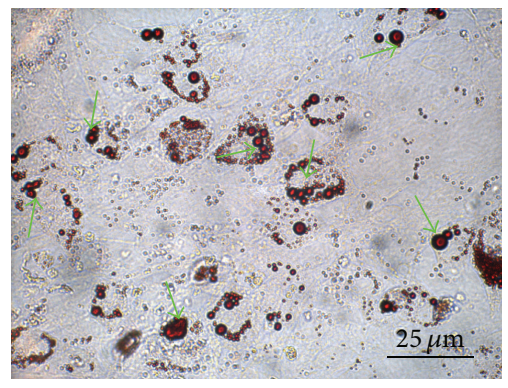

(b)

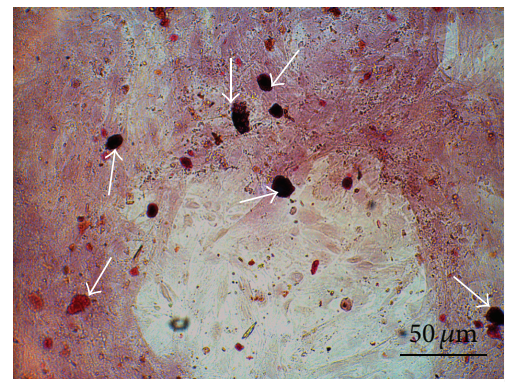

(c)

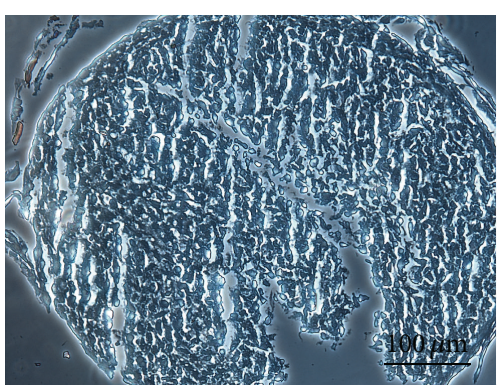

(d)

FIGURE 2: Characterization of human bone marrow-derived stem cells coexpressing luciferase and green fluorescent protein reporter genes (Luc-GFP-hBMSCs). (a) Flow cytometric analysis of Luc-GFP-hBMSCs. Luc-GFP-hBMSCs were harvested with $0.25 \%$ trypsin-EDTA and resuspended in phosphate-buffered saline supplemented with $2 \%$ fetal bovine serum. Following this, the expression levels of CD90, CD105, CD29, CD73, CD44, CD34, CD45, CD11c, and HLA-DR were measured by fluorescence-activated cell sorting analysis. (b)-(d) Luc-GFPBMSCs were able to differentiate into adipocytes, osteoblasts, and chondrocytes in vitro as shown by positive Oil Red O staining (b), Alizarin red staining (c), and Alcian Blue staining (d). The green arrows indicate lipid droplets stained with Oil Red O, and the white arrows indicate calcium nodules stained with Alizarin red. Acid mucopolysaccharide of chondrocytes could be stained by Alcian Blue. 

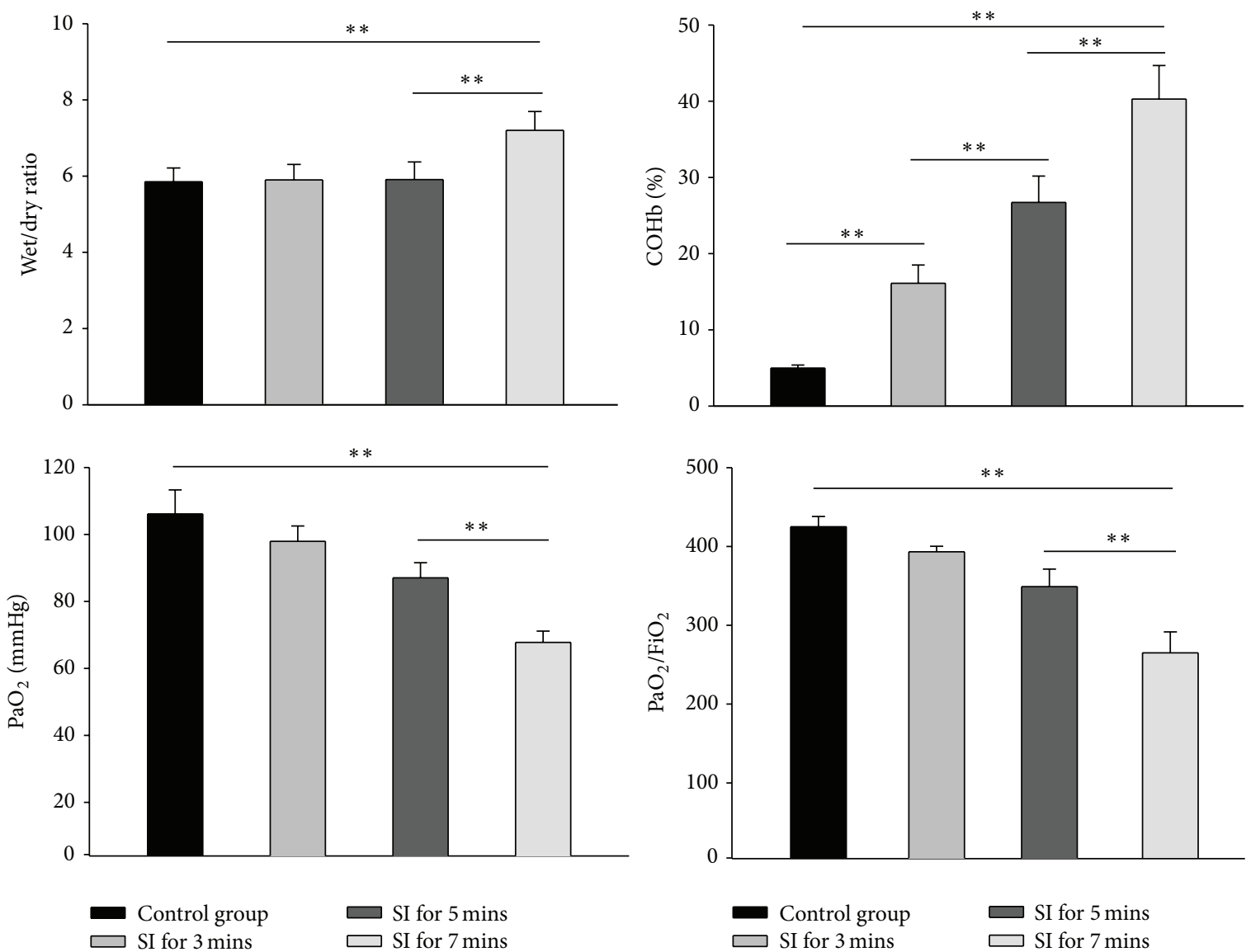

(a)
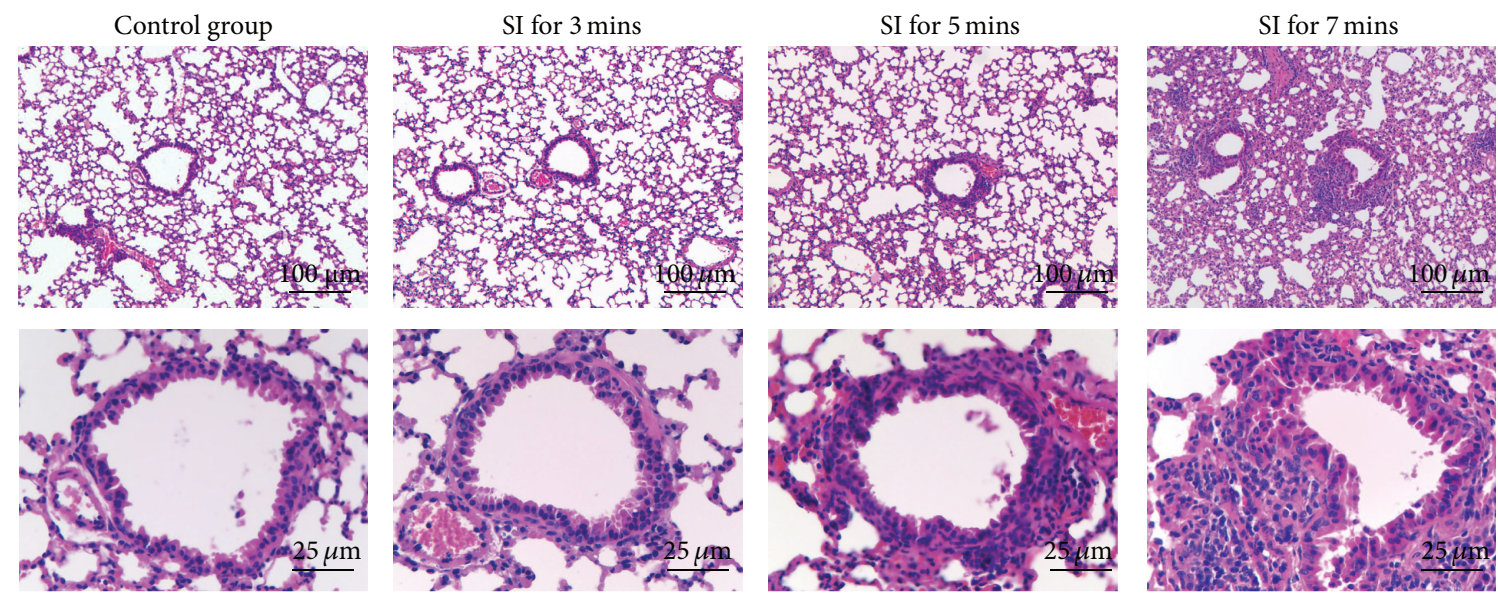

(b)

FIGURE 3: Establishment of smoke inhalation NOD/SCID mouse model. NOD/SCID mice $(n=6)$ were subjected to $0,3,5$, 7, and 9 min of smoke exposure. (a) Wet/dry (W/D) weight ratios, blood carboxyhemoglobin $(\mathrm{COHb}), \mathrm{PaO}_{2}$, and $\mathrm{PaO}_{2} / \mathrm{FiO}_{2}$ were measured at the indicated time points. $* *$ indicates $P<0.01$ between the indicated groups. (b) Representative histological images of lung sections from mice with or without smoke inhalation injury $(n=6)$. 

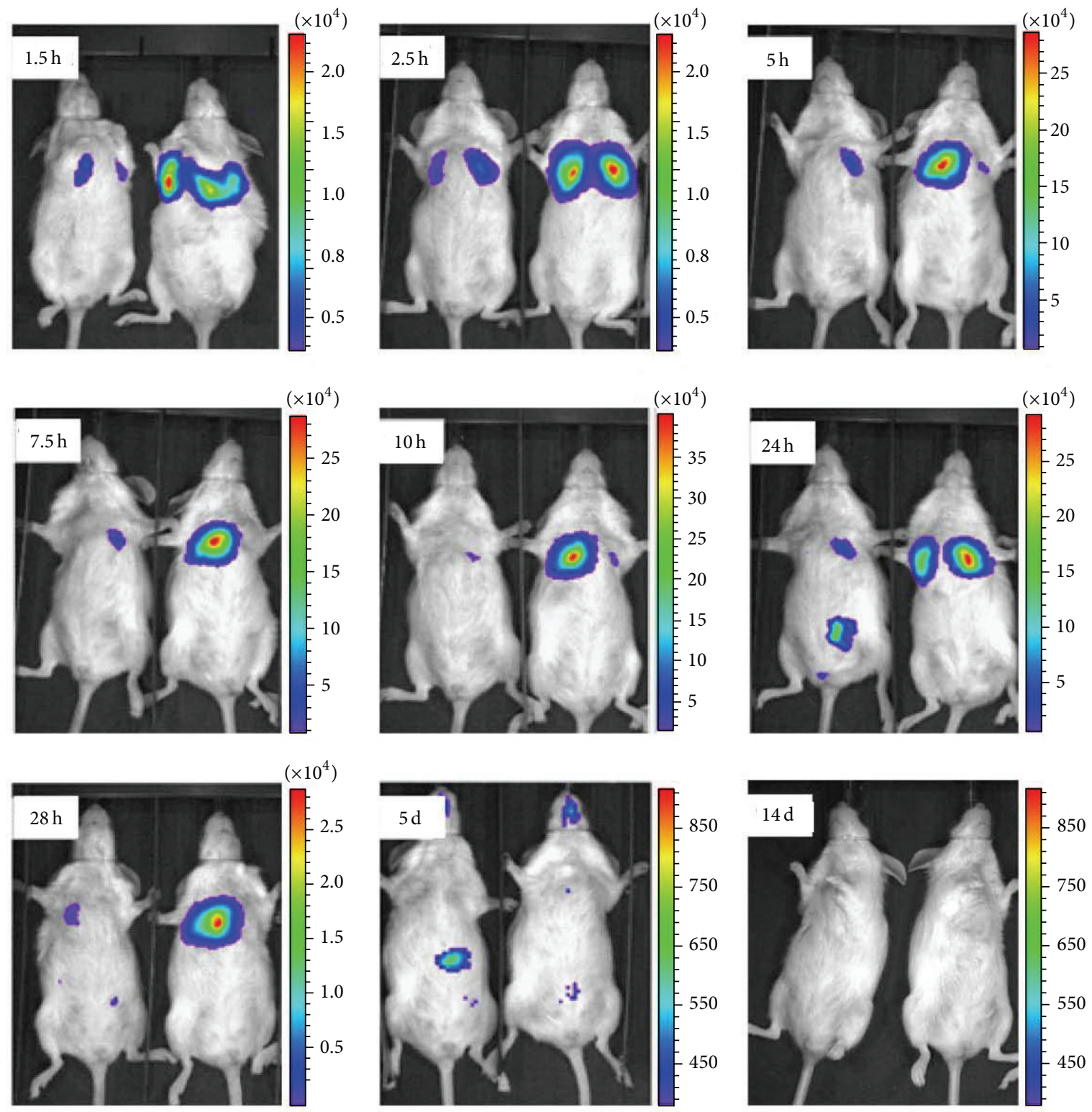

(a)

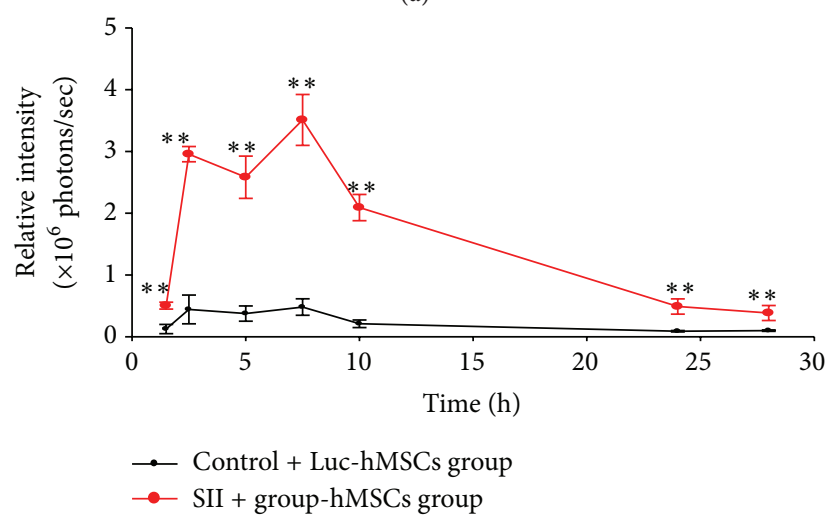

(b)

FIGURE 4: Biodistribution of human bone marrow-derived stem cells coexpressing luciferase and green fluorescent protein reporter genes in mice with or without smoke inhalation injury. Human bone marrow-derived stem cells coexpressing luciferase and green fluorescent protein reporter genes (Luc-GFP-hBMSCs) were intravenously injected into NOD/SCID mice and imaged at 1.5, 2.5, 5, 7.5, 10, 24, and 28 h and 5 and $14 \mathrm{~d}$ after injection. (a) The Luc-GFP-hBMSCs initially localized to the lung and then migrated to the abdomen by $24 \mathrm{~h}$. Reporter gene expression completely disappeared by $14 \mathrm{~d}$ after injection. (b) Quantification of the bioluminescent signal over this time period indicated that a greater number of systemically infused Luc-GFP-hBMSCs localized to the lungs in mice with smoke inhalation injury compared to uninjured mice $(n=6) . * *$ indicates $P<0.01$ compared with the uninjured control group treated with Luc-GFP-hBMSCs. 


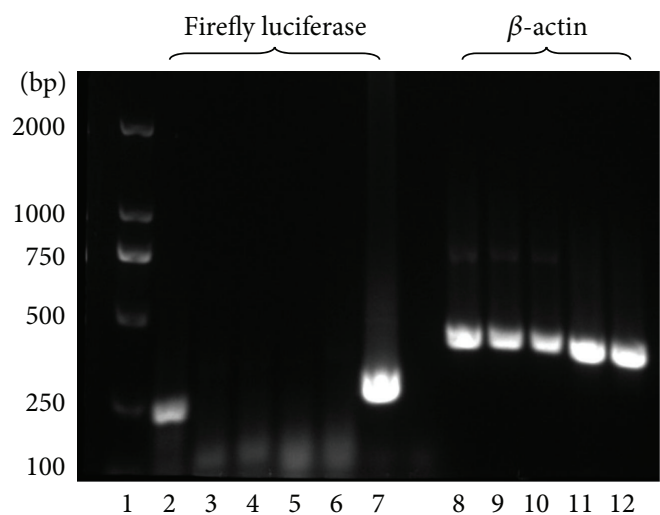

(a)

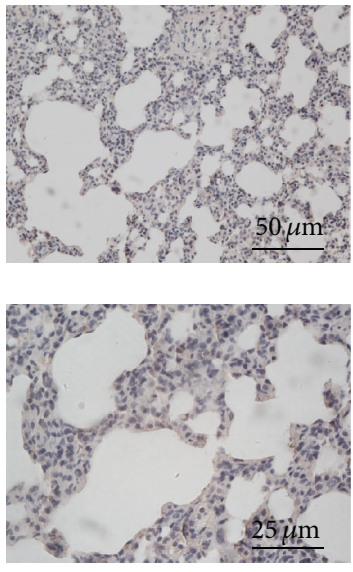

Control + Luc-hMSCs group
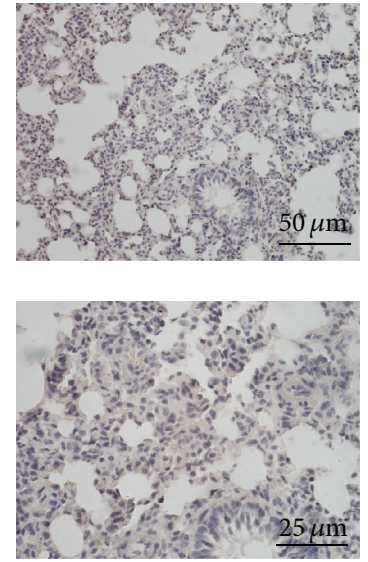

SII + Luc-hMSCs group

(b)

FIGURE 5: Detection of human bone marrow-derived stem cells coexpressing luciferase and green fluorescent protein reporter genes in mouse tissues at 14 days after injection. (a) Expression of the firefly luciferase gene was detected by PCR. $\beta$-actin was used as a loading control. Panel 1: DNA marker. Panels 2-7: from left to right, human bone marrow-derived stem cells coexpressing luciferase and green fluorescent protein reporter genes (Luc-GFP-hBMSCs), liver from a control mouse treated with Luc-GFP-hBMSCs, liver from a mouse with smoke inhalation injury (SII) treated with Luc-GFP-hBMSCs, lung from a control mouse treated with Luc-GFP-hBMSCs, and lung from a mouse with SII treated with Luc-GFP-hBMSCs. Panels 8-12: corresponding $\beta$-actin levels from panels 2-6. Representative images from at least three independent experiments. (b) Luc-GFP-hBMSCs were detected by immunohistochemistry (anti-GFP) in the lung at day 14 after injection into mice with SII $(n=6)$.

the culture supernatant. Moreover, 1 day after injection of hBMSCs, the levels of KGF in BALF were increased compared with control and SII groups $(P<0.05)$ (Figure $7(\mathrm{a})$ ).

MSC could downregulate expressions of proinflammatory factors to protect the host from extraordinary inflammatory damage [18]. Our results found that systemic treatment with hMSCs could significantly decrease the levels of TNF- $\alpha$ in BALF (Figure 7(b)), which may contribute to downregulating inflammatory responses and tissue injury.

\section{Discussion}

In China, ALI caused by smoke inhalation is the most common cause of death among victims of fire tragedies. At least $85 \%$ of deaths from fire disasters occur because of excessive inhalation of smoke and toxic gases [28]. Characterized by acute onset, rapid progression, severe illness, and high mortality, severe respiratory disease resulting from smoke inhalation is commonly seen in clinical practice [29].

ALI is a severe pathological condition clinically characterized by respiratory distress, refractory hypoxemia, and noncardiogenic pulmonary edema. A number of factors can lead to the development of ALI; these include sepsis, pneumonia, trauma, aspiration of gastric contents, and exposure to large amounts of smoke from fires [30]. Smoke inhalation-induced ALI has unique pathophysiological features that differ from ALI caused by sepsis or pneumonia. Components found within smoke, including particulate materials, systemic toxins, and respiratory irritants, trigger the production of a cascade of inflammatory mediators within the airway mucosa and lung parenchyma, causing damage to mucosal lining and leading to peribronchial inflammation, which ultimately can result in pulmonary edema and ventilation/perfusion mismatch [1, 3-5]. During this process, intrapulmonary leukocyte aggregation following activation of the classic complement cascade releases even more chemokines and cytokines, leading to airway cast formation and widespread plugging. Moreover, the resultant induction of nitric oxide (NO) synthase in respiratory epithelial cells and alveolar macrophages leads to NO production, which increases bronchial blood flow, decreases hypoxic pulmonary vasoconstriction in poorly ventilated regions within the lung, and results in ventilation/perfusion mismatch. $\mathrm{NO}$ also forms peroxynitrite $\left(\mathrm{ONOO}^{-}\right)$by combining with superoxide $\left(\mathrm{O}_{2}{ }^{-}\right)$ produced by neutrophils, which can lead to DNA damage and alveolar epithelial cell death $[4,5]$.

As with the other factors that cause ALI, efficient and specific therapies are needed for smoke inhalation-induced ALI. To develop these therapies, additional studies are needed because the pathological mechanisms underlying ALI remain poorly understood, and the current supportive methods used to treat the condition, including basic mechanical ventilation, fluid resuscitation, and oxygen administration, are not as effective as desired $[1,4,6]$. Different types of cell therapies are expected to have the ability to cure a wide variety of diseases, substantially improving the routine therapies currently used in the clinic [31]. Due to their low expression levels of immune antigens, MSCs are an attractive cell resource for the treatment of various complicated and refractory diseases [32]. Preclinical studies in small (mouse and rat) and large (sheep) animal models, as well as ex vivo studies using perfused human lungs, have demonstrated the potential 


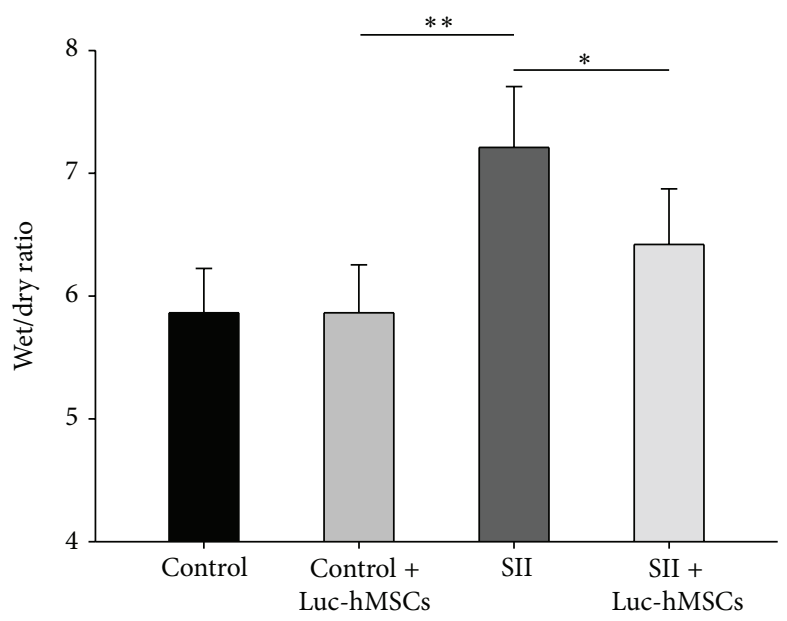

(a)

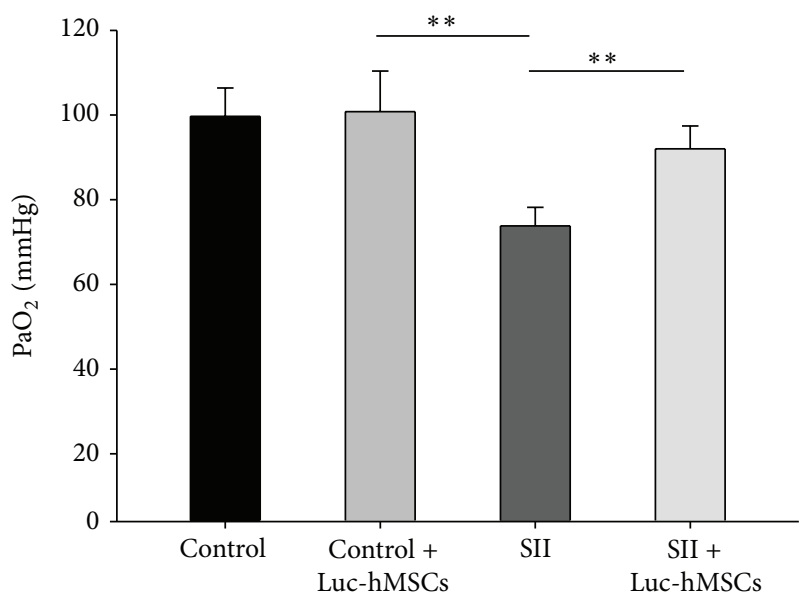

(b)

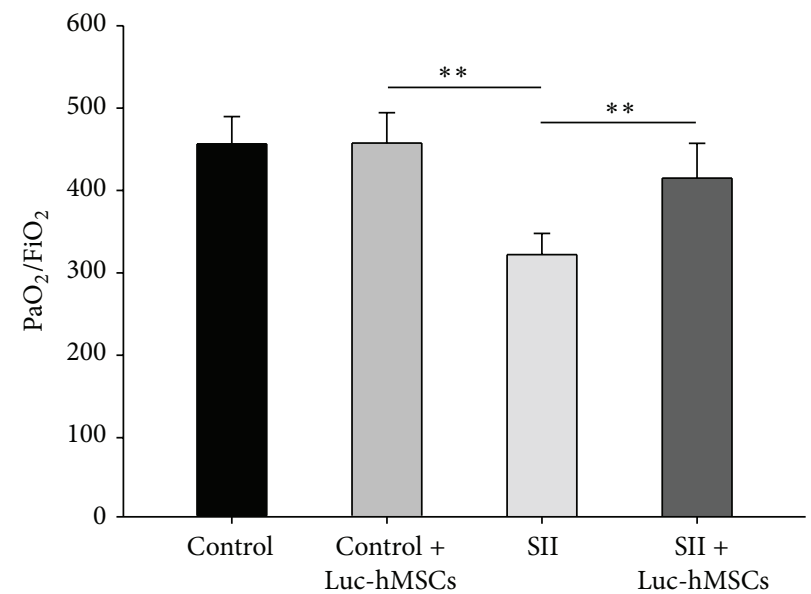

(c)
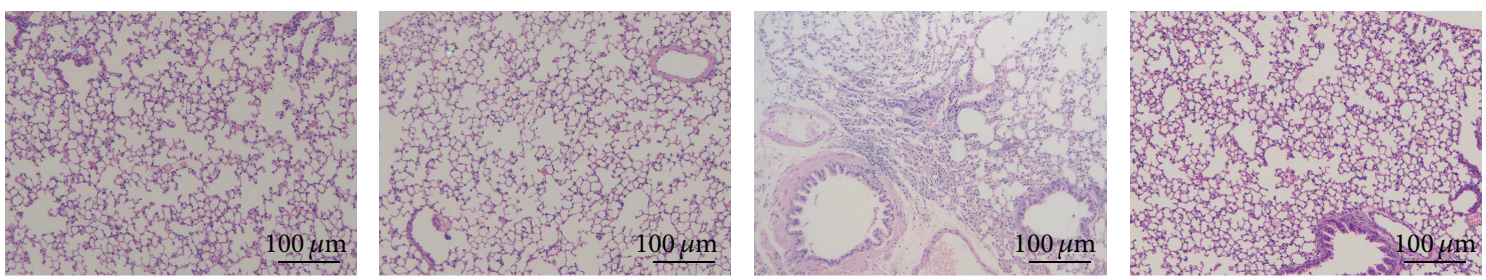

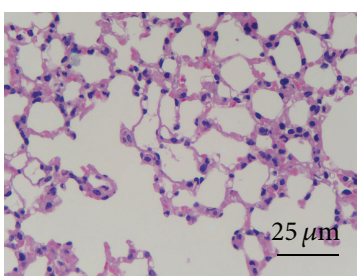

Control group

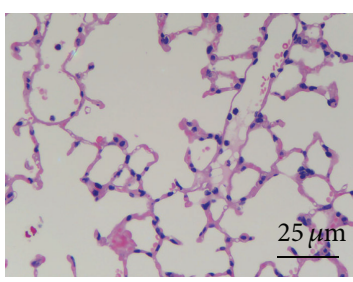

Control + Luc-hMSCs group

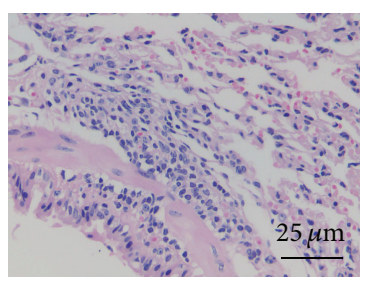

SII group

(d)

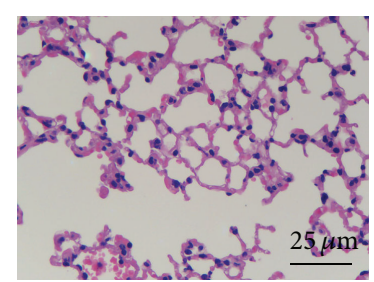

SII + Luc-hMSCs group

FIGURE 6: Evaluation of the protective effects of hMSCs against smoke inhalation lung injury in mice. (a)-(c) Wet/dry (W/D) weight ratios, $\mathrm{PaO}_{2}$, and $\mathrm{PaO}_{2} / \mathrm{FiO}_{2}$ were measured at 14 days after injection of hBMSCs into mice with or without SII $(n=6) .{ }^{*} P<0.05$, ${ }^{* *} P<0.01$ between the indicated groups. (d) Representative pictures of histological examinations of lung sections from the indicated groups. 


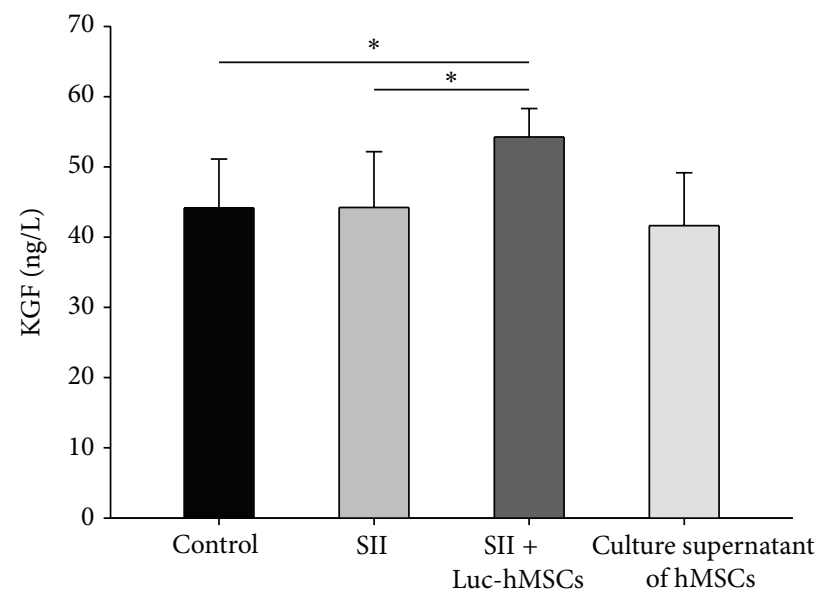

(a)

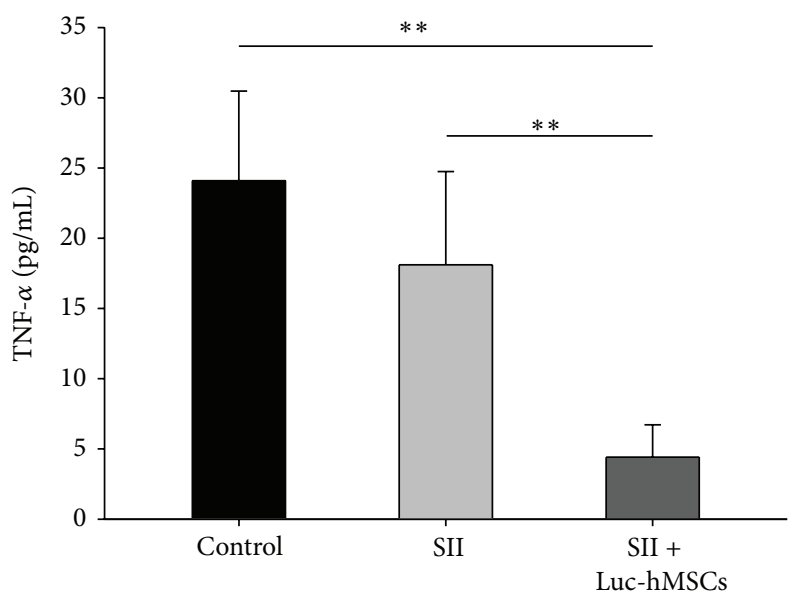

(b)

FIgURE 7: The effects of hMSCs treatment on the levels of TNF- $\alpha$ and KGF in BALF of mice with SII. At one day after injection of hMSCs, (a) the concentrations of keratinocyte growth factor (KGF) in the BALF were measured for control, SII, and Luc-hMSCs treatment groups. The culture supernatant of hBMSC was used as a positive control. (b) The concentrations of TNF- $\alpha$ in the BALF were measured for the indicated groups $(n=6) .{ }^{*} P<0.05,{ }^{* *} P<0.01$ between the indicated groups.

efficacy and safety of MSC administration for the treatment of ALI/ARDS [17-20,33]. In the current study, we demonstrated that hMSCs exert a protective effect on mice with smoke inhalation-induced ALI, suggesting that these cells may offer a therapeutic strategy for SII.

Despite recent interest in the use of adult stem cell therapy due to the multipotent nature of bone marrow-derived stem cells, findings regarding the engraftment process of systemically administered hBMSCs in lung injury models have varied. The majority of studies on experimental lung injuries have demonstrated an MSC engraftment rate of less than 1$5 \%$ [34-36]. In the current study, using BLI, we tracked the distribution dynamics of systemically administered hBMSCs in immunodeficient mice with SII. The results showed that a greater number of hBMSCs were found in the lung tissues of SII mice compared with control mice, a finding that is similar to previous studies [21] of mice exposed to trauma or bearing tumors. Our results further confirmed that hBMSCs innately traffic to sites of inflammation, which are concordant with previous studies of MSC localization patterns in trauma, cancer, and following exposure to radiation [37-39]. However, we found no hBMSC engraftment in the lung, which was demonstrated by the complete disappearance of BLI signal at 14 days after hBMSC administration. This result indicates that engraftment of the cells into the lung is not a major driving force behind the beneficial effects that were noted.

Growing evidence has indicated that the effects of MSCs with regard to lung tissue repair are not attributable to the differentiation capacity of these cells but rather to their activation of a protective mechanism and their stimulation of endogenous regeneration factors $[18,40]$. MSCs produce soluble bioactive factors known to reduce alveolocapillary membrane permeability, inhibit apoptosis and fibrosis, decrease inflammation, and enhance tissue repair. Impaired AFC (alveolar fluid clearance) is common in patients with ALI/ARDS and leads to pulmonary edema. BMSCs also produce several epithelial growth factors, including vascular endothelial growth factor (VEGF), keratinocyte growth factor (KGF), and hepatocyte growth factor (HGF). KGF has been shown to improve alveolar fluid transport in part by upregulating aENaC gene expression [41] and $\mathrm{Na}-\mathrm{K}-\mathrm{ATPase}$ activity [42]. As such, KGF is important in the resolution of lung injury. In a set of ex vivo experiments, Matthay MA and colleges found that siRNA-mediated inhibition of KGF expression decreased the beneficial effects of MSCs in restoring AFC in injured, perfused human lungs by approximately $80 \%$ [19]. In the current study, we found that MSCs significantly attenuated pulmonary edema induced by smoke inhalation. The paracrine secretion of KGF leading to the restoration of AFC may be a possible mechanism for this finding.

The immunomodulatory effects of MSCs are wellestablished, and MSCs exert protection against inflammatory damage by downregulating the expression of proinflammatory factors, such as IL-1b, IL-8, interferon- (INF-) $\gamma$, and TNF- $\alpha$ [43]. MSCs also secret anti-inflammatory agents such as IL-4 and IL-10 to regulate the development of lung inflammation [44], reduce neutrophil infiltration into the lung, and reduce the quantity of proinflammatory cytokines in circulation, collectively maintaining a balance between inflammatory and anti-inflammatory responses [45]. Our results showed that hMSCs could significantly decrease the levels of TNF- $\alpha$ in BALF after 1 day after injection. Correspondingly, smoke inhalation resulted in inflammatory cell infiltration around the airway, which was significantly attenuated by the administration of hBMSCs. Thus, immunomodulation plays an important role in downregulating inflammatory responses and attenuating tissue injury in SII.

In summary, our study demonstrated that systemically administered hBMSCs mainly localized to the lungs of mice with SII. The hBMSCs attenuated the lung injury induced by smoke inhalation, and this may be due to the effect of 
anti-inflammatory and secreting KGF of hMSCs but not associated with the differentiation potential of the cells.

\section{Competing Interests}

The authors declare that they have no competing interests.

\section{Authors' Contributions}

MeiJuan Song and Qi Lv contributed equally to this paper.

\section{Acknowledgments}

This research was partially supported by the National Natural Science Foundation of China (81202105 and 71533008) and the Tianjin Science and Technology Project (15JCYBJC28500, 13JCQNJC12900-2013, and 14ZCDZSY00033). The authors appreciate the help provided by the Chinese Academy of Medical Sciences and the Medical University of Tianjin's basic medical school.

\section{References}

[1] R. H. Demling, "Smoke inhalation lung injury: an update," Eplasty, vol. 8, article e27, 2008.

[2] D. L. Smith, B. A. Cairns, F. Ramadan et al., "Effect of inhalation injury, burn size, and age on mortality: a study of 1447 consecutive burn patients," Journal of Trauma-Injury, Infection and Critical Care, vol. 37, no. 4, pp. 655-659, 1994.

[3] E. C. Kimmel and K. R. Still, "Acute lung injury, acute respiratory distress syndrome and inhalation injury: an overview," Drug and Chemical Toxicology, vol. 22, no. 1, pp. 91-128, 1999.

[4] M. H. Toon, M. O. Maybauer, J. E. Greenwood, D. M. Maybauer, and J. F. Fraser, "Management of acute smoke inhalation injury," Critical Care and Resuscitation, vol. 12, no. 1, pp. 53-61, 2010.

[5] M. O. Maybauer, S. Rehberg, D. L. Traber, D. N. Herndon, and D. M. Maybauer, "Pathophysiology of acute lung injury in severe burn and smoke inhalation injury," Anaesthesist, vol. 58, no. 8, pp. 805-812, 2009.

[6] The Chinese Medical Association, Clinical Diagnosis and Treatment Guidelines: Burns Booklet, 2007.

[7] N. Nugent and D. N. Herndon, "Diagnosis and treatment of inhalation injury," Total Burn Care, vol. 3, pp. 262-272, 2007.

[8] B. J. Jones and S. J. Mctaggart, "Immunosuppression by mesenchymal stromal cells: from culture to clinic," Experimental Hematology, vol. 36, no. 6, pp. 733-741, 2008.

[9] M. R. Reagan and D. L. Kaplan, "Concise review: Mesenchymal stem cell tumor-homing: detection methods in disease model systems," Stem Cells, vol. 29, no. 6, pp. 920-927, 2011.

[10] M. Introna, G. Lucchini, E. Dander et al., "Treatment of graft versus host disease with mesenchymal stromal cells: a phase I study on 40 adult and pediatric patients," Biology of Blood and Marrow Transplantation, vol. 20, no. 3, pp. 375-381, 2014.

[11] I. B. Resnick, C. Barkats, M. Y. Shapira et al., "Treatment of severe steroid resistant acute GVHD with mesenchymal stromal cells (MSC)," American Journal of Blood Research, vol. 3, no. 3, pp. 225-238, 2013.

[12] J. Bartunek, A. Behfar, D. Dolatabadi et al., "Cardiopoietic stem cell therapy in heart failure: The C-CURE (cardiopoietic stem cell therapy in heart failURE) multicenter randomized trial with lineage-specified biologics," Journal of the American College of Cardiology, vol. 61, no. 23, pp. 2329-2338, 2013.

[13] S. Forostyak, P. Jendelova, and E. Sykova, "The role of mesenchymal stromal cells in spinal cord injury, regenerative medicine and possible clinical applications," Biochimie, vol. 95, no. 12, pp. 2257-2270, 2013.

[14] M.-E. M. Amer, S. Z. El-Sayed, W. A. El-Kheir et al., "Clinical and laboratory evaluation of patients with end-stage liver cell failure injected with bone marrow-derived hepatocyte-like cells," European Journal of Gastroenterology and Hepatology, vol. 23, no. 10, pp. 936-941, 2011.

[15] A. Skrahin, R. K. Ahmed, G. Ferrara et al., "Autologous mesenchymal stromal cell infusion as adjunct treatment in patients with multidrug and extensively drug-resistant tuberculosis: an open-label phase 1 safety trial," The Lancet Respiratory Medicine, vol. 2, no. 2, pp. 108-122, 2014.

[16] J. G. Wilson, K. D. Liu, H. Zhuo et al., "Mesenchymal stem (stromal) cells for treatment of ARDS: a phase 1 clinical trial," The Lancet Respiratory Medicine, vol. 3, no. 1, pp. 24-32, 2015.

[17] G. Yin, G. Hu, R. Wan et al., "Role of bone marrow mesenchymal stem cells in L-arg-induced acute pancreatitis: effects and possible mechanisms," International Journal of Clinical and Experimental Pathology, vol. 8, no. 5, pp. 4457-4468, 2015.

[18] J. W. Lee, X. Fang, A. Krasnodembskaya, J. P. Howard, and M. A. Matthay, "Concise review: mesenchymal stem cells for acute lung injury: role of paracrine soluble factors," Stem Cells, vol. 29, no. 6, pp. 913-919, 2011.

[19] M. A. Matthay, B. T. Thompson, E. J. Read et al., "Therapeutic potential of mesenchymal stem cells for severe acute lung injury," Chest, vol. 138, no. 4, pp. 965-972, 2010.

[20] J. W. Lee, X. Fang, N. Gupta, V. Serikov, and M. A. Matthay, "Allogeneic human mesenchymal stem cells for treatment of E. coli endotoxin-induced acute lung injury in the ex vivo perfused human lung," Proceedings of the National Academy of Sciences of the United States of America, vol. 106, no. 38, pp. 16357-16362, 2009.

[21] S. Kidd, E. Spaeth, J. L. Dembinski et al., "Direct evidence of mesenchymal stem cell tropism for tumor and wounding microenvironments using in vivo bioluminescent imaging," STEM CELLS, vol. 27, no. 10, pp. 2614-2623, 2009.

[22] D. Zhao, Y. Tu, L. Wan et al., "In vivo monitoring of angiogenesis inhibition via down-regulation of Mir-21 in a VEGFR2-luc murine breast cancer model using bioluminescent imaging," PLoS ONE, vol. 8, article e71472, 2013.

[23] F. Zhu, X. Qiu, J. Wang et al., "A rat model of smoke inhalation injury," Inhalation Toxicology, vol. 24, no. 6, pp. 356-364, 2012.

[24] R. Yu, X. Guo, L. Huang, Z. Zeng, and H. Zhang, "The novel peptide PACAP-TAT with enhanced traversing ability attenuates the severe lung injury induced by repeated smoke inhalation," Peptides, vol. 38, no. 1, pp. 142-149, 2012.

[25] S. Hou, H. Ding, Q. Lv et al., “Therapeutic effect of intravenous infusion of perfluorocarbon emulsion on LPS-induced acute lung injury in rats," PLoS ONE, vol. 9, no. 1, Article ID e87826, 2014.

[26] S. Bhaumik, X. Z. Lewis, and S. S. Gambhir, "Optical imaging of Renilla luciferase, synthetic Renilla luciferase, and firefly luciferase reporter gene expression in living mice," Journal of Biomedical Optics, vol. 9, no. 3, pp. 578-586, 2004.

[27] V. M. Ranieri, G. D. Rubenfeld, B. T. Thompson et al., "Acute respiratory distress syndrome: the Berlin definition," The Journal 
of the American Medical Association, vol. 307, no. 23, pp. 25262533, 2012.

[28] L. Yan and H. J. Fan, "Advances in bone marrow mesenchymal stem cells in the treatment of acute lung injury," Chinese Journal of Emergency Medicine, vol. 21, pp. 328-331, 2012.

[29] S. Lei, L. Daibo, and C. Jia, "Advances in drug therapy for acute smoke inhalation induced lung injury," Chinese Journal of Asthma, vol. 4, pp. 292-297, 2011.

[30] L. B. Ware and M. A. Matthay, "The acute respiratory distress syndrome," The New England Journal of Medicine, vol. 342, no. 18, pp. 1334-1349, 2000.

[31] A. Tyndall, "Mesenchymal stem cell treatments in rheumatology: a glass half full?" Nature Reviews Rheumatology, vol. 10, no. 2, pp. 117-124, 2014.

[32] F. Gao, S. M. Chiu, D. A. Motan et al., "Mesenchymal stem cells and immunomodulation: current status and future prospects," Cell Death and Disease, vol. 7, no. 1, article e2062, 2016.

[33] S. Asmussen, H. Ito, D. L. Traber et al., "Human mesenchymal stem cells reduce the severity of acute lung injury in a sheep model of bacterial pneumonia," Thorax, vol. 69, no. 9, pp. 819825,2014

[34] N. Gupta, X. Su, B. Popov, J. W. Lee, V. Serikov, and M. A. Matthay, "Intrapulmonary delivery of bone marrow-derived mesenchymal stem cells improves survival and attenuates endotoxin-induced acute lung injury in mice," Journal of Immunology, vol. 179, no. 3, pp. 1855-1863, 2007.

[35] M. Rojas, J. Xu, C. R. Woods et al., "Bone marrow-derived mesenchymal stem cells in repair of the injured lung," American Journal of Respiratory Cell and Molecular Biology, vol. 33, no. 2, pp. 145-152, 2005.

[36] D. N. Kotton, A. J. Fabian, and R. C. Mulligan, "Failure of bone marrow to reconstitute lung epithelium," American Journal of Respiratory Cell and Molecular Biology, vol. 33, no. 4, pp. 328334, 2005.

[37] H. Wang, F. Cao, A. De et al., "Trafficking mesenchymal stem cell engraftment and differentiation in tumor-bearing mice by bioluminescence imaging," Stem Cells, vol. 27, no. 7, pp. 1548$1558,2009$.

[38] M. Studeny, F. C. Marini, J. L. Dembinski et al., "Mesenchymal stem cells: potential precursors for tumor stroma and targeteddelivery vehicles for anticancer agents," Journal of the National Cancer Institute, vol. 96, no. 21, pp. 1593-1603, 2004.

[39] A. H. Klopp, E. L. Spaeth, J. L. Dembinski et al., "Tumor irradiation increases the recruitment of circulating mesenchymal stem cells into the tumor microenvironment," Cancer Research, vol. 67, no. 24, pp. 11687-11695, 2007.

[40] K.-Y. Yang, H.-C. Shih, C.-K. How et al., "IV delivery of induced pluripotent stem cells attenuates endotoxin-induced acute lung injury in mice," Chest, vol. 140, no. 5, pp. 1243-1253, 2011.

[41] Y. Wang, H. G. Folkesson, C. Jayr, L. B. Ware, and M. A. Matthay, "Alveolar epithelial fluid transport can be simultaneously upregulated by both KGF and $\beta$-agonist therapy," Journal of Applied Physiology, vol. 87, no. 5, pp. 1852-1860, 1999.

[42] B. P. H. Guery, C. M. Mason, E. P. Dobard, G. Beaucaire, W. R. Summer, and S. Nelson, "Keratinocyte growth factor increases transalveolar sodium reabsorption in normal and injured rat lungs," American Journal of Respiratory and Critical Care Medicine, vol. 155, no. 5, pp. 1777-1784, 1997.

[43] M. Hayes, G. Curley, B. Ansari, and J. G. Laffey, "Clinical review: stem cell therapies for acute lung injury/acute respiratory distress syndrome-hope or hype?" Critical Care, vol. 16, article 205, 2012.
[44] Z.-X. Liang, J.-P. Sun, P. Wang, Q. Tian, Z. Yang, and L.-A. Chen, "Bone marrow-derived mesenchymal stem cells protect rats from endotoxin-induced acute lung injury," Chinese Medical Journal, vol. 124, no. 17, pp. 2715-2722, 2011.

[45] S. S. Iyer and M. Rojas, "Anti-inflammatory effects of mesenchymal stem cells: novel concept for future therapies," Expert Opinion on Biological Therapy, vol. 8, no. 5, pp. 569-581, 2008. 

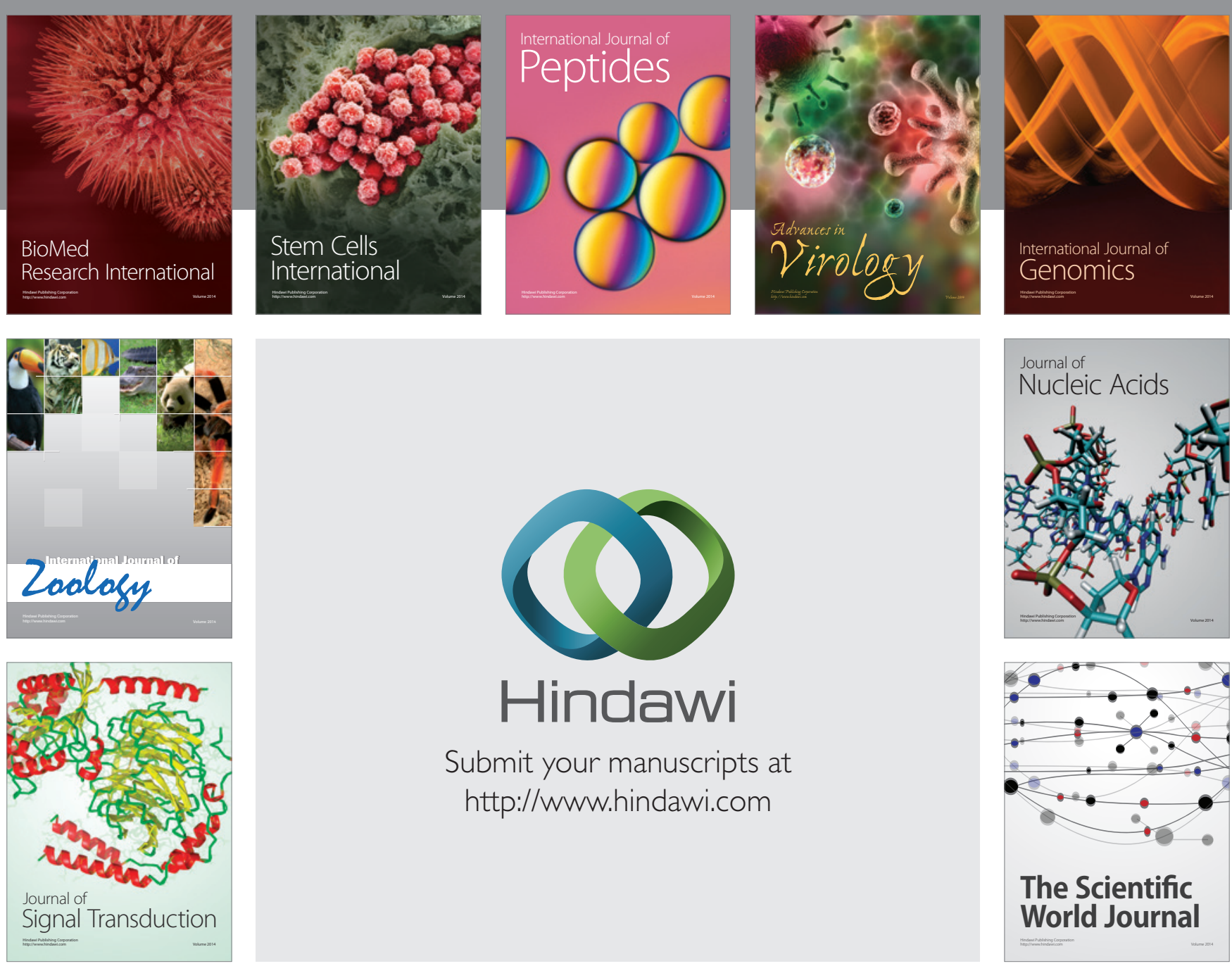

Submit your manuscripts at

http://www.hindawi.com
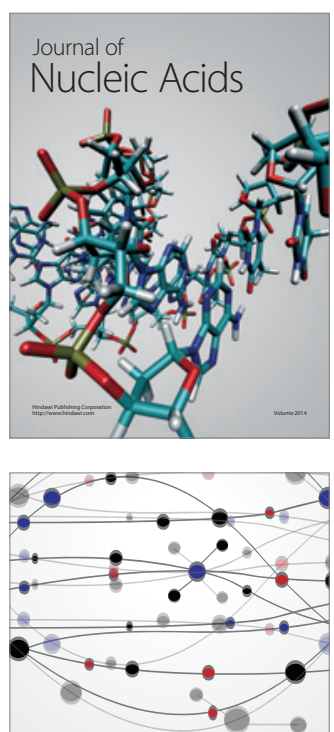

The Scientific World Journal
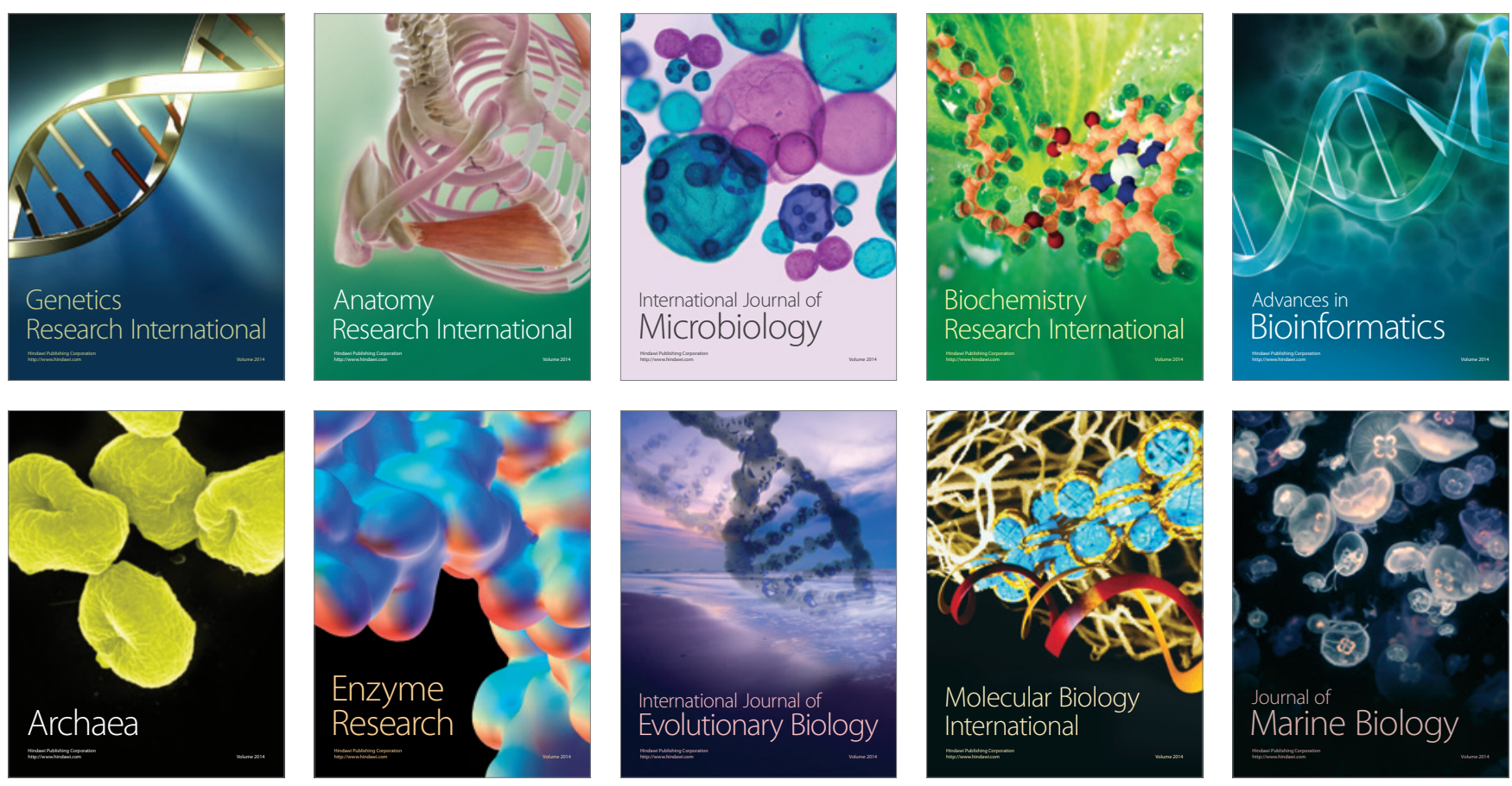